\title{
Le « corroborée » du rugby languedocien n'est plus que légende
}

Jean-Bernard Marie Moles

\section{(2) OpenEdition \\ 1 Journals}

\section{Édition électronique}

URL : http://journals.openedition.org/corpsetculture/591

DOI : 10.4000/corpsetculture.591

ISSN : $1777-5337$

\section{Éditeur}

Association Corps et Culture

\section{Édition imprimée}

Date de publication : 1 juin 1999

ISSN : 1268-5631

\section{Référence électronique}

Jean-Bernard Marie Moles, "Le " corroborée » du rugby languedocien n'est plus que légende », Corps et culture [En ligne], Numéro 4| 1999, mis en ligne le 24 septembre 2007, consulté le 08 septembre 2020. URL : http://journals.openedition.org/corpsetculture/591 ; DOI : https://doi.org/10.4000/ corpsetculture.591

Ce document a été généré automatiquement le 8 septembre 2020.

(c) tous droits réservés 


\title{
Le « corroborée » du rugby languedocien n'est plus que légende
}

\author{
Jean-Bernard Marie Moles
}

Introduction

1 «Le rugby est une légende qui ne vit que de rites et de mythes ». C'est un « ancien » au plus profond de l'histoire d'Ovalie, qui m'a fait découvrir la liminarité de ce sport collectif. Mais c'est dans les « lieux et moments » (Turner V., 1969) que j'ai pu, par ma situation privilégiée de pratiquant puis de reporter, appréhender la secondarité, celle où se délivrent, certes parcimonieusement, « les dissonances entre l'identité sociale et l'investissement affectif » (Rémy J. et Voyé L., 1974) et comprendre la force latente des rites dans notre Rugby d'Oc (languedocien). Cet article sera donc une réflexion tirée d'un positionnement emic ${ }^{1}$, donc relaté à l'intérieur du système selon la définition de Turner (1982), ayant pour référent l'acteur rugbystique, ce combattant sorti du routinier.

2 L'angle de cette analyse sera défini notamment à partir des rites de passage dans un monde conventionnel et fédéraliste. Ces rites de passage se formulant dans un ensemble d'actions fortement codifiées, qui tendront à démunir le rugbyman de toutes ses références emmagasinées dans la quotidienneté, jusqu'à provoquer une action d'inversion, afin qu'il côtoie le monstrueux et le fantastique, les pratique et les disserte. Bref qu'il se dépouille de ses valeurs journalières, construites en fonction d'une logique rationnelle et abstraite, pour d'autres plus coercitives, élaborées à partir de situations contextuelles et d'un climat émotionnel éprouvants.

3 Ce qui va nous intéresser dans cette approche empirique c'est la dramaturgie que les interactants rugbystiques écrivent dans ces rites et ses effets qui peuvent conduire l'épique à devenir inacceptable. Les actions rituelles rugbystiques seront ainsi approfondies dans un contexte événementiel, productrices de signification mais aux conséquences irréversibles, car plus seulement intangibles comme le supposent les légendes, mais bien tangibles comme l'exprime mon expérience d'observateur attentif et averti.

Le contexte du rituel rugbystique 
4 La tourmente mercantile qui s'est abattue sur le rugby à XV français, provoquant une césure irréparable entre le rugby de haut-niveau, à échelle européenne et nationale et celui qualifié d'inférieur par les plus outranciers - en fait il s'agit du rugby de séries régionales, donc à l'échelle régionale et elle seule -, continue d'épandre depuis 1995 ses terribles effets pervers. Quand les chaînes télés se repaissent de rencontres élitistes, le rugby de clocher, des villages, des bourgades crie famine devant la désaffection des supporters et du public, eux-mêmes désarçonnés par la disparition de clubs illustres et mythiques, qui pour survivre s'unissent dans des ententes nébuleuses où l'âme s'égare dans des sigles " sécurité sociale "2, provoquant perplexité et confusion car point n'est aisé pour les ennemis d'hier de s'acoquiner avec les amis d'aujourd'hui.

Le Rugby d'Oc (languedocien) voguait en 1980 avec 90 clubs et leur authentique culture, de conquêtes en triomphes, de titres en «faits divers » malséants, de derbys bellicistes en vénération de héros totémiques. Vingt ans plus tard, avec ses 49 clubs dont 17 ententes à deux, voire trois ou quatre clubs, il pleure son passé héroïque et populaire, observe ses stades désertés et la passion éventée, a peine à oublier ses joutes dominicales qui n'existent plus que dans les mémoires et constate l'émergence d'une nouvelle culture, hétérogène, où la somme des clochers est moins que la religion d'Ovalie, avec un football prédominant, usurpateur du lien social et qui fait du Rugby d'Oc une pratique délitescente.

6 Le Rugby d'Oc, dans son épanouissement, se lisait dans sa ritualité la plus féconde : celle du combat, de l'affrontement. Cette ritualité qui invite tous les dimanches à $15 \mathrm{~h}$, un groupe de pacifistes de la quotidienneté à se faire face, se combattre, se culbuter, se piétiner, couler sang et hurler de douleur (ce qu'ils évitent avec un art consommé de la prévention dans cette quotidienneté).

7 Ce «peuple d'Ovalie », comme le qualifie le Président du Comité du Languedoc Françis Sénégas, "est garant d'une socialité particulière car nouée par un sentiment d'appartenance prégnant à une même cause $»^{3}$ (tant d'ailleurs chez les pratiquants que les supporters) : défendre le maillot, son village donc faire preuve d'amour et de fierté, de courage et d'altruisme. Il navigue entre processus émotionnels et affectuels, "baigné dans une tribu, dans une communauté " ${ }^{4}$ et " poussé par une raison sensible, qui s'enrichit du contraire» (Maffesoli M., 1997), soit sortir temporairement d'une société individualiste, anonyme, morose et pacifiste, pour s'immiscer dans une autre, communautaire, populaire, conviviale et vindicative.

8 Ce temps cyclique, passer du normal à l'anormal, oppose assurément le sens à la signification. Le rituel du rugby, se retrouver en tribu et éprouver toutes sortes de rites, d'initiés et secrets, de passage et de transcendance ne peut pas se restreindre au sens tribal et communautaire, mais il peut révéler une forte signification, soit d'un grand désœuvrement, notamment social, soit d'une roborative motivation à rompre avec la routine quotidienne par une confluence de volontés et s'affirmer être différent de ce qu'impose la société. Sinon comment comprendre les trois rites majeurs et pérennants du rituel rugbystique que sont :

- la préparation d'avant-match, psychique et mentale.

- le choc des premières mêlées fermées.

- La 3e mi-temps.

9 Rites de "contre-interaction» (Goffman E., 1988) pour les deux premiers, car en oxymore avec les règles de politesse, les gestes usuels protocolaires, où il ne s'agit plus de «rites d'évitement» mais plutôt de «rites de confrontation» qui concourent 
d'ailleurs à une rationalité de transgression du bienséant (Picard D., 1996) ${ }^{5}$ et à une justification de "profanations rituelles ». Rite festif pour le 3e, avec l'instauration de l'harmonie après la dysharmonie. Ces rites de "contre-éthique" (pour les deux premiers), à l'encontre des «rites qui sont des règles de conduite qui prescrivent comment l'homme doit se comporter avec les choses sacrées» (Durkheim E., 1912) renvoient à une communion au schéma sacrificiel, aux cérémonies sacrées claniques.

Le rugby se nourrit en fait de « rites de passage " (Van Gennep, 1909), rites successifs et concomitants qui marquent pour les rugbymen un changement d'appartenance d'un milieu social à une communauté secrète et restrictive, changements accoudés encore à ceux liés aux corps, engobés de vaseline, ceints d'élastoplast, de genouillères, coudières, épaulières, casques et protège-dents. Le citoyen paisible part au combat et se prépare en tant que tel, avec ces changements liés au sacré et à la symbolique (transgression des limites de l'entendement pour côtoyer la transcendance, la folie même comme nous le verrons dans les chapitres suivants, avec une tendance à la martyrisation, au sacrificiel).

11 La césure avec le monde profane est irréversible dans le rituel du rugby, certes dans une temporalité brève et effrénée, mais d'une très forte intensité émotionnelle, au point d'ailleurs que les dérapages peuvent être fréquents et graves! «On parle beaucoup de rites dans le rugby, souligne Francis Sénégas, le Président du Comité du Languedoc, mais en fait je crois que certains confondent la sacralisation de sentiments collectifs, avec une intoxication psychique, un bourrage de crâne, lesquels conduisent à des transcendances graves, transcendances que j'appellerai sommairement "pétage de plombs". Quand on voit sortir des joueurs la bave aux lèvres, prêts à tuer, excusez-moi, mais ces rites de passage d'un monde à un autre, je m'en passerai bien $»^{6}$.

À l'orée de l'an 2000, la lutte contre les rites rugbystiques, en particulier ceux « d'avantmatch » et "des premières mêlées fermées" a dans l'élite largement porté ses fruits, avec un jeu certes toujours plus engagé mais désormais plus noble et la tempérance qui s'impose. Au niveau régional, cette lutte à dominer la transcendance, rendue nécessaire devant les écarts de conduite, a connu hélas de nombreux effets perfides, - analysés plus loin - qui atténuent les mythèmes du Rugby d'Oc, avec une passion raisonnée, peu féconde de références mythiques et dont s'accommode peu ce peuple d'Ovalie, gavé durant des décennies d'une théâtralisation qui tirait sa sève dans ces rites. Le Rugby d'Oc a-t-il ainsi perdu de son aura en galvaudant ses rites? Les rites valent-ils mieux que le jeu? Le jeu du rugby sans ses rites ancestraux peut-il être encore différent des autres disciplines collectives?

13 Voyons comment l'évolution des trois rites majeurs du rugby à XV dans notre Languedoc, a pu avoir de fortes répercussions dans la contextualisation de ce sport jusqu'alors le plus populaire dans notre région, sur sa prépondérance sociale et sa perception par les médias.

Le rite d'avant-match

14 L'avant-match est dans tous les sports collectifs un instant de communion. Une communauté se retrouve, se forme, pour faire face à l'enjeu et à l'adversaire. Le collectif, l'esprit d'équipe supplante l'individu. L'entraîneur va s'atteler à souder son groupe, pour ne faire qu'un. Dans bon nombre de sports collectifs, celui-ci devra certes s'attacher à motiver ses joueurs, mais il devra aussi prendre garde à leur garder lucidité, préserver leur compétence, leur maîtrise et pratique. Dans les vestiaires d'une équipe de rugby, cet instant de recherche d'harmonie est majoré par le dessein de 
sceller pas seulement les hommes, mais leur volonté de se surpasser dans le combat (le rugby n'est pas un sport d'opposition mais de confrontation).

Le rite identitaire, avec la remise des maillots aux couleurs du club, de la région, symbole de l'esprit de territorialité -, le brassard de capitaine, est toujours teinté de gravité, voire de solennité. Le citoyen « rugbyman » doit admettre qu'il est maintenant un «soldat ». Un soldat qui va monter en première ligne, partir au front, se révéler courageux, valeureux et fier de la relique emblématique qu'il a endossée (l'amour du maillot). Mais cette raison guerrière n'est pas suffisante. Il faut aller au-delà. Atteindre le sublime, le titanesque et le transcendant.

Cette phase dite "d'action réorientée» (Turner, 1982), qui se situe entre la marginalisation du lieu sacré qu'est le vestiaire (réservé aux seuls officiants) et l'agrégation solennelle de la communauté à s'élever (d'être la meilleure), propose à chacun des équipiers un changement de statut. Avec l'attribution de surnoms symboliques (pour exemples, un 2e ligne de Valras était surnommé Le Mammouth, d'autres Le Chameau, L'Enclume, Le Thon, La Braise, La Mule, à Palavas il y avait La «Terrible » Cigogne, Alain Estève à Béziers c'était Le Grand, Roques à Cahors, Le Roc, Doménech à Brive, Le Duc, Patrick Estève à Narbonne, Le TGV) ou de surnoms restrictifs (Califano à Toulouse c'est Cali, N'Tamack c'est Milou, Cantoni à Béziers était Canto, Cabrol... Riton, Saisset... Olive, Codorniu à Narbonne... Codor).

Puis existe la partition entre les fantassins (les avants) et les cavaliers (les trois-quarts). Tous sont soldats, mais les fantassins iront au feu en premier. Voilà pourquoi ils sortent rarement s'échauffer : «Il faut les garder au chaud, dans la pénombre des vestiaires, comme des Miuras. Pour qu'ils rugissent et crachent leur venin quand s'ouvrira la porte des vestiaires et monteront les clameurs $~{ }^{7}$. "L'initiation guerrière " représente ici, effectivement, "une transformation mémorable et va requérir une fidélité et une confiance totale aux normes de la communauté où les postulants sont introduits " (Rivière C., 1996). Transformation qui s'effectue dans une cérémonie forte émotionnellement, avec son langage propre, souvent châtié. Qui peut tourner encore à l'obsession "piaculaire", se sacrifier pour réparer une défaillance, une faute, éventuelle ou virtuelle.

18 Ainsi se forge un mental de guerrier en piétinant l'un de ses partenaires sur le sol même des vestiaires (il faut se montrer sans pitié), en se fracassant la tête contre les murs ou la porte des vestiaires pour exorciser sa peur (le courage doit être sans faille), se martyrisant pour être prêt à recevoir le mal (être héroïque). Cette obsession de la transcendance s'appuie aussi sur l'humiliation : « N'être qu'une merde, qu'un chieur de colle, qu'un porteur de couches $\|^{8}$ ne peut que provoquer rage et désespérance. Un entraîneur d'une sélection française n'hésitait pas à cracher sur le visage de ses joueurs pour leur faire comprendre qu'ils n'étaient « rien » et qu'il attendait d'eux qu'ils soient « tout ». Le rien c'est la honte, le tout c'est l'honneur. Le choix était frugal mais vital.

Dans ce rite d'avant-match, le surpassement de soi n'est que la prémisse de la transe. Cette transe qui va conduire des joueurs à fouler aux pieds leur entraîneur qui s'est mis en travers de la porte des vestiaires, leur hurlant que "rien ne doit les arrêter ", à déchiqueter une paillasse portant le maillot de l'équipe adverse, à cracher ou uriner sur des articles de presse vexatoires, ou encore Claude Spanghero, capitaine du grand Narbonne de 1981, qui avait enfermé ses troupes dans les vestiaires avant la demi-finale à Montferrand et jeté les clés par la fenêtre en clamant : "Si vous voulez gagner ce 
match, il va falloir sortir d'ici ». Et ses hommes de s'exécuter en défonçant la porte et ses montants à grands coups d'épaule et de cris rageurs9.

Mais cette sacralisation de la transe et du sacrificiel peut vite verser dans l'irréversible, le consternant, l'affligeant, tant d'ailleurs au plus haut-niveau (rappelons-nous comment les avants du XV français, lors d'un test match à Nantes, avaient tranché dans un piétinement collectif et sauvage, le sexe de Wayne Shelford, le capitaine All-Balck) ${ }^{10}$ qu'au niveau régional, avec des entames de matches plus proches de rixes que de jeux de ballons. Cependant si, grâce à la télévision (notamment avec des caméras dans les vestiaires, véritable sacrilège pour les coutumiers des rites rugbystiques), au plus haut niveau, ce rite d'avant-match s'est largement atténué pour éviter que la spectacularisation attendue sur le petit écran ne devienne une contre-publicité, en louant la modération au surpassement, au niveau du rugby régional le détachement à la transcendance, métamorphose rituelle, n'a pu se faire que par la législation.

En effet devant les effets pernicieux de ce rituel de passage du citoyen au guerrier, le Comité du Languedoc a décidé à partir des années 90 d'imposer aux équipes de se préparer et de s'échauffer dans leur composante totale en dehors des vestiaires, dans l'un des en-but et d'y rester jusqu'au coup d'envoi. L'intimité, le secret étant proscrits, le rite d'avant-match se trouva démuni de ses valeurs nourricières de transe. Le rugby y gagna de la sérénité et de la loyauté, mais perdit la finalité de ce rite, l'explosion de guerriers transcendés par « toucher l'impossible ». Les débuts de match sont désormais paisibles. Les arbitres s'en font une joie, mais le public a semble-t-il du mal à apprécier ces nouvelles conduites. Les traditions des « 5 premières minutes de boîtes à gifles » ne sont plus aujourd'hui dans notre Rugby d'Oc qu'un mythe.

Le rite de la première mêlée

Pour les premières lignes aux oreilles en "chou-fleur", sceau indéfectible de leur appartenance à la tribu des piliers, celle des «tronchards", blessures guerrières qui valident leur courage et leur vaillance, la ritualité du combat s'ébauche dans le rite des premiers chocs tête contre tête quand l'arbitre ordonne le premier affrontement réglé (la mêlée fermée). Ce rite du " choc de crânes vaselineux » est partagé par chaque tribu, équipe. C'est l'instant de vérité, qui donne signification aux équipiers. Si dans ce premier affrontement, preuve est faite que reculer n'est pas jouer et avancer c'est gagner, alors la transcendance va gagner l'ensemble de l'équipe. Ce culte épicrânien des "tronchards", est une symbolique forte pour le moral d'une équipe. Et le bruit "sourd» du choc des crânes va déclencher un lien le plus souvent capital pour le prolongement du match entre ceux qui vont «à la mine », se ruent dans ce maelström d'hommes où se brisent les plus fortes convictions, et la "cavalerie légère ", les demis et trois-quarts, dont la sensation appétitive résulte de l'émotion de ce premier affrontement.

Aujourd'hui, notamment dans les divisions nationales, ce rite de la première mêlée se déroule dans les règles légitimes, soit avec force et vigueur, roublardise et vice, mais sans violence illégitime. Ce qui fût longtemps la panacée du rugby d'avant l'apophtegme du 21 août 1995, quand le rugby devint professionnel et se dût de convaincre les télévisions qu'il était un sport spectaculaire et pas seulement empreint de violence bâtarde. Car auparavant le rite de la première mêlée se doublait de ce que les puristes appellent « une mêlée relevée ». Effectivement, précédant le heurt barbare crânien, deuxième partie du rite, les « tronchards » entamaient le rituel du combat par une débauche de coups de poings et de coups de pied à la volée qui faisait rarement de 
dégâts substantiels. Mais il s'agissait de donner une orientation implacable à l'adversaire, soit pour ceux qui jouaient à domicile : « on est à la maison et les patrons c'est nous », soit pour les visiteurs « on se cassera pas, on n'a peur de rien ».

L'émotion des belligérants résultait toujours de la perception de changements corporels et faciaux des adversaires à la mêlée suivante. Instant sacré où dans les comportements expressifs, notamment sur le visage, se lit la peur ou la confiance : « Tu savais comment allait être le match ", souligne Christian Tralléro, champion de France en 1979 avec le R.C. Narbonne, «ce rite de la première mêlée était accepté par tous, même ceux qui savaient qu'ils avaient plus à y perdre qu'à y gagner ${ }^{11}$. Ce rite n'avait pour but que de créer une peur instantanée, mais si le but n'était pas atteint, le respect devenait mutuel.

Un rite créateur de lien social, d'osmose, âme de tribalité

Cette première mêlée était très attendue par le public et quand parfois elle était provoquée volontairement (en envoyant au coup d'envoi le ballon directement en touche), un long murmure montait des tribunes et des gradins. Tout le monde savait que c'était un instant de vérité et qu'il n'y avait point de dérobade. Chacun acquiesçait à ce rite sans pitié, qu'il soit confiant ou non. Mais il était accepté sans restriction. Aujourd'hui ce rite a moins de signification, même s'il garde toute sa valeur émotionnelle, car dans le rugby d'élite les caméras des télévisions traquent sans atermoiement les "violents» et dans le rugby régional, les sanctions sont désormais implacables si par malheur ce rite débouche dans une violence aveugle.

L'atténuation des portées émotionnelles de ce rite fait partie assurément, même d'une manière minime, de la déshérence actuelle du public vis-à-vis du rugby régional, car dans la spectacularisation celui-ci ne peut prétendre rivaliser avec le rugby élitaire. La fonctionnalité du rugby régional était d'établir un lien éminemment affectif entre l'équipe et son village. Les supporters et le public moins averti, aimaient à se reconnaître dans ce rite de la première mêlée qui présageait du match et qui d'ailleurs, quand les «tronchards » se neutralisaient trop souvent pouvait perdurer : «T'as vu ils ne sont pas partis ", « ils sont gaillards les nôtres, on a peur de rien », « On va vous en mettre plein la gueule ».

Le lien entre l'initié et le regardant se formalisait sans détour, si bien que le public se transcendait autant que ses joueurs: "Quand ça chauffait, les supporters étaient comme des maquisards qui sortaient de la forêt. Ils venaient en fait en renfort. On était tous sur la brèche $»^{12}$. Et le " on» résume parfaitement cette osmose. Les réponses émotionnelles des supporters relevaient de programmes affectifs propres à la violence mentale de ce rite. La connivence affective se fondait aussi dans un contexte particulier, et se révélait d'autant plus exaltante qu'il s'agissait de derbys.

Rite de reconnaissance pour les regardants

Ce rite de la première mêlée, rite d'appartenance d'abord à la tribu des "tronchards ", rite de passage dans la réalité du match, passer du statut de pacifiste à celui de guerrier, de pragmatique à fantastique, était ainsi un rite de reconnaissance pour les regardants, qui ne l'étaient plus seulement et in fine participaient de facto à l'accomplissement et l'aboutissement du rite. Ce rite devait impérativement pour les supporters participer à leur besoin d'appartenance à une même tribu. Las, le polissage du rite a eu raison de l'engouement à cette adhésion du Rugby d'Oc, qui peine à se ressourcer et retrouver une autre vitalité. 
29 La contestation fédéraliste aux rites, au niveau régional, a ralenti le consensus social entre club et village. Ce qui n'avait finalement aucune finalité utilitaire et rationnelle, apparaît cependant aujourd'hui comme rassembleur, unificateur, fédérateur. Et si ce rite s'avère toujours autant irrationnel, voire incongru, car comment expliquer que des pacifistes deviennent guerriers, jouent du « bourre-pif $»^{13} 80$ minutes durant, avant de s'embrasser et fêter leur transcendance bras dessus, bras dessous durant la $3 \mathrm{e}$ mitemps, son étiolement consumant la légende dans des braises homériques, plonge la théâtralité de cette culture languedocienne qu'est le rugby, dans le commun de la banalité. Il n'est jamais bon que le rugby coutumier se fasse fonctionnel, car la légende nourrit les souvenirs. Et vivre sur ses souvenirs n'a jamais été un signe de prospérité.

Le rite de la $3^{\mathrm{e}}$ mi-temps

30 Aujourd'hui les derbys endiablés, où chaque village venait célébrer dans la fête et la ferveur, sa culture, celle d'un rugby de corsaires pour les clubs pescaïres (Valras, Portla-Nouvelle, Palavas), celle d'un rugby courage pour les clubs de vignerons (Coursan, StAndré, Sérignan, Sigean, Argeliers, Salles d'Aude), celle d'un rugby volontariste et solidaire pour les clubs de gavachs ${ }^{14}$ du Gévaudan (Mende, St-Affrique), de la HauteVallée (Couiza, Espéraza, Quillan, Pays de Sault), des montagnards (des Cévennes : Bédarieux, St-Pons) se perd dans les méandres de l'histoire du Rugby d'Oc, qui si riche fût-elle n'offre plus qu'un présent morose.

31 Ces différences étaient la composante de la culture holiste du rugby languedocien, culture qui trouvait sa sève dans le courage, la vaillance et l'excellence. Un multi tribalisme qui s'identifiait dans une composante globaliste, le rugby d'Occitanie et qui, par ses outrances ou ses instants homériques, écrivait l'histoire d'une culture, celle du Rugby d'Oc.

32 Dans cette mythologie rugbystique languedocienne, le rituel tenait une part cruciale. Les rites étant considérés comme une praxéologie, comme un mode de communication très particulier, décuplant la fascination de l'appartenance tribale et la symbolique du chevalier Bayard à partir, comme le souligne Radcliffe-Brown : « de l'émulsion d'un sentiment d'insécurité et de danger » (1952) qui transcendait les acteurs et pouvait rendre selon Francis Sénégas, président du Comité du Languedoc : «le plus rationnel des joueurs totalement irrationnel, le plus docile en fauve carnassier ${ }^{15}$. Vilfredo Pareto y aurait distingué " une action non logique quoique pleine de sens » (1916). Comme peut l'être encore la $3 \mathrm{e}$ mi-temps, instant privilégié où partenaires et adversaires s'unissent autour d'un verre, n'oubliant point la lutte guerrière à peine évanouie, mais la commentant, la louant, couvrant d'éloges ceux qu'ils agonisaient d'injures et de menaces, dans une mêlée cette fois-ci conviviale et festive.

On retrouve dans ce rite de la 3e mi-temps, la distinction que Marcel Mauss et Émile Durkheim avançaient entre les rites positifs, avec l'implication d'actes prescrits comme un rassemblement amical, une cérémonie consensuelle, et les rites négatifs, c'est-à-dire interdits (symbolique de l'anthropophagie par exemple, souvent évoquée dans les avant-matchs « on va les bouffer, les déchirer, les cuire à petit feu, leur ouvrir le ventre, la tronche, leur péter la gueule, les embrocher... " ${ }^{16}$. Dans ces 3 e mi-temps, il ne s'agit point pour les combattants fourbus de se mettre en valeur, ou "garder la face » (Goffman E., 1994) mais dans une sorte de commémoration, de mettre en évidence l'existence d'un consensus autour d'idéaux communs et se convaincre, quoique de tribus différentes, d'appartenir à une même communauté. 

quotidienneté que le surpassement de soi portant sur le précédent (rites d'avant-match et des mêlées fermées). Étrange contexte que ce rite de la 3e mi-temps où doit être louée l'intégration sociale, alors qu'auparavant, pour les mêmes acteurs étaient prônées l'exclusion et la destruction. Dans cette fusion, partenaires, adversaires, dirigeants et supporters (parfois même arbitres), apparaît sans détour leur complémentarité, qui n'est in fine que le drame qu'écrivent les joueurs en le pratiquant, puis en le décrivant. La 3e mi-temps c'est l'épique contingent à l'homérique : «Les légendes, prétendent les anciens, naissent plus souvent dans ce rite de la 3e mi-temps que sur les prés $»^{18}$. Cette $3 e$ mi-temps est une forte composante symbolique du rituel rugbystique, car elle démontre combien les interactants, après une défenestration mentale, sont des personnes dignes de respect.

cestentonique, est porteur cependant d'une tradition rétrograde car machiste (seulement ouvert aux hommes). Quoique désuète, cette restrictivité a largement participé à la mythologie du rugby, « un sport d'hommes », " il faut des couilles dans le short », «c'est pas un sport de gonzesses $~^{19}$, mais il s'avère aujourd'hui que cette discrimination ne pourra perdurer plus longtemps, notamment face à l'émergence du rugby féminin et son absolue nécessité (pour pallier l'absence d'équipes de jeunes, rendues obligatoires par la FFR, celle-ci a autorisé les clubs à aligner des équipes féminines, de façon à éviter une rétrogradation d'office) ${ }^{20}$. Serait-ce la fin de ce rite ? Pour Radcliffe-Brown : «l'évolution repose sur un processus de diversification qui fait apparaître des structures sociales de plus en plus complexes » (1994).

Les femmes aux banquets officiels, au cœur des 3es mi-temps, l'avant-match qui se prépare en régional non plus dans la pénombre et le secret des vestiaires mais au grand jour, en public, sur le pré, ou dans l'élite sous le regard croisé de caméras indiscrètes, des mêlées ordonnées assagies, avec des tronchards casqués pour se préserver d'oreilles bourgeonnantes, oui le corroborée des indigènes languedociens a bien perdu de son authenticité.

\section{BIBLIOGRAPHIE}

Durkheim E. (1994) Les Formes élémentaires de la vie religieuse, Paris, PUF. (1912).

Goffman E. (1974) Les Rites d'interaction, Paris, Minuit.

Goffman E., (1987) Façons de parler, Paris, Minuit. (1981).

Maffesoli M. (1997) Du nomadisme, Paris, Le Livre de poche.

Moles J.B. (1997) Mémoire de Maîtrise : Ordre et désordre dans l'émergence du professionnalisme dans le rugby français, Montpellier, Université Montpellier, Faculté des Sciences du Sport (Directeur de recherche Y. Le Pogam).

Pareto V. (1968) Traité de sociologie générale, Genève, Droz. (1916).

Picard D. (1996) Les Règles du savoir-vivre, Sciences Humaines, 58, p28. 
Radcliffe Brown A.R. (1968), Method in Social Anthropology, Chicago, Srinivas (1968).

Radcliffe Brown A.R. (1994) Structure et fonction dans la société primitive de Karl M. Van Meter (dir) in La Sociologie, Paris, Larousse, 369-415 (1952).

Rémy J. et Voyé. L (1974). La Ville et l'urbanisation, Duculot. Cité par Leclerq E. (1992) Du Rituel à Théâtralité. Une lecture de Victor Turner, Cahiers Internationaux de Sociologie, XCII, 181-198.

Rivière C. (1996) Les Cérémonies profanes, Sciences Humaines, 58, p27.

Turner V. (1969) Le Phénomène rituel, Paris, PUF.

Turner V. (1982) From ritual to theatre, the human seriousness of play, New-York, PAJ.

Van Gennep A. (1981), Les Rites de passage, Picard (1909).

\section{NOTES}

1. Terme utilisé la première fois par Turner V. (cité par Leclerq, note 26, p191) - voir biblio -. Le point de vue Emic est celui de l'indigène, qui définit lui-même un objet.

2. Exemples : l'Entente Servian-Boujan (l'ESB), l'Entente Vendres-Sauvian-Lespignan (L'EVSL), l'Entente Sigean-La Nouvelle (L'ESLN)...

3. Sénégas Fr., Président du Comité du Languedoc, Entretien pour thèse (1999).

4. Conférence de presse Michel Maffesoli, 19 Janvier 1999, Montpellier.

5. «Consiste en un ensemble de modèles comportementaux et de prescriptions régissant les interactions sociales ».

6. Op. cit. p3.

7. Brunel Cl., entraîneur de Poussan.

8. Winterstein Al., ex-capitaine de Bédarieux.

9. Journal Du Dimanche, 28/02/1999.

10. Rugbyrama, 1986.

11. Tralléro Christian, champion de France avec Narbonne en 1979, puis Président Joueur du C.A. Bédarieux, champion de France de 3e division. Entretien pour thèse, Moles J.B. (1999).

12. Op. cit p6.

13. Expression populaire typiquement rugbystique, largement employée dans le rugby des séries régionales languedocien.

14. Terme populaire employé par les pescaïres, qui désigne les paysans.

15. Op. cit. p3.

16. Expressions récurrentes dans les avant-matchs.

17. Inquiétude, angoisse qui trouve sa réponse avec le stress, dont l'utilisation commune a été dans l'hexagone inversée.

18. Op. Cit. p.4.

19. Expressions populaires.

20. La Fédération Française de Rugby à XV obligeait jusqu'alors tout club affilié à aligner des équipes jeunes, exclusivement masculines. Depuis deux ans, un club peut éviter toute sanction en suppléant aux équipes masculines, une équipe féminine. En 1999, le club audois de Leucate a usé de cette nouvelle législation. 


\section{RÉSUMÉS}

Dans le rituel du rugby qu'est le combat, trois rites majeurs se déclament dans une temporalité et théâtralité récurrentes, l'avant-match (la préparation psychologique), le moment du match (avec les premiers affrontements en mêlées ordonnées) et l'après-match (la 3e mi-temps). Dans ces rites de passage du citoyen paisible au combattant impitoyable et dans une récursivité étonnante, $\mathrm{du}$ guerrier vaincu ou vainqueur au tout un chacun durant la $3 \mathrm{e}$ mi-temps, le surpassement du soi, l'exaltation des sentiments collectifs, du sublime et du titanesque dans une débauche sacrificielle, ne cessent d'impliquer, comme le soutenait Vilfredo Pareto (1916), les interactants dans « une action non logique quoique pleine de sens pour celui qui l'accomplit». C'est non seulement à la compréhension de ce sens que cette recherche s'est attachée, mais encore à l'évolution de ces rites face à la demande et aux contraintes sociales rapportées au rugby languedocien : un rugby populaire plutôt qu'élitiste.

In the fight which is the ritual of the rugby, three major rites spout in a repeated temporality; before the match (psychological training), during the match (with the first confrontations in methodical scrums) and after the match (the «third half-time»). In those passing rites, from the peaceful citizen to the pitiless fighter and in an astonishing return during the third half-time from the defeated or victorious warrior to the common run of people, the surpassing of oneself, the exalting of collective feelings, some sublime and some titanic in a sacrificial excess, all roated in an intense notion of initiatory secret, though methodically repeated in a closed temporality, haven't stopped involving, as Vilfredo Pareto was stating (1916), the actors in «a no logical action though full of meaning for the one who is performing it». It is to the understanding of this meaning that this research deals with, but also to the evolution of these rites facing the social demand and constraints; particularly the rugby from Languedoc, the popular one rather than the elitist one.

\section{INDEX}

Keywords : rite, ritual, rugby, transcendence

Mots-clés : rituel, transcendance

\section{AUTEUR}

\section{JEAN-BERNARD MARIE MOLES}

Groupe de Recherche en Information et Communication (GRIC). Université Montpellier I. Équipe « Corps et Culture». 